\title{
RESULTADOS LABORATORIAIS COMPARATIVOS EM FÊMEAS NELORE DE DIFERENTES CATEGORIAS PROVENIENTES DE FAZENDAS COM E SEM A PRESENÇA DE Pteridium arachnoideum NAS PASTAGENS
}

André Hiroshi Quadros Watanabe ${ }^{1}$ Wilmar Sachetin Marçal ${ }^{2}$

WATANABE, A. H. Q.; MARÇAL, W. S. Resultados laboratoriais comparativos em fêmeas Nelore de diferentes categorias provenientes de fazendas com e sem a presença de Pteridium arachnoideum nas pastagens. Arq. Ciênc. Vet. Zool. UNIPAR, Umuarama, v. 19, n. 1, p. 5-10, jan./mar. 2016.

\begin{abstract}
RESUMO: A Pteridium arachnoideum, anteriormente classificada como Pteridium aquilinum, popularmente conhecida por samambaia, é um dos vegetais tóxicos mais preocupantes em vários países, incluindo o Brasil. No estado do Paraná há várias regiões onde a planta possui característica endêmica. Pesquisadores brasileiros caracterizaram, experimentalmente, três formas clínicas das doenças causadas pela samambaia aos bovinos: diátese hemorrágica, hematúria enzoótica dos bovinos e carcinomas epidermoides do trato digestório superior. No presente trabalho, estudaram-se os valores hematológicos, bioquímicos e de urinálise em duas diferentes fases do desenvolvimento animal, sendo 58 novilhas e 55 vacas, originadas de propriedades rurais com características zootécnicas semelhantes e com a presença ou não da samambaia. A finalidade foi estabelecer um protocolo para triagem e adotar medidas na melhoria do manejo, evitando-se mais prejuízos. Neste estudo, houve uma queda no número de hemácias de vacas e novilhas provenientes de propriedades rurais com presença de samambaia. $\mathrm{O}$ número de plaquetas foi significativamente superior em ambas as faixas etárias nos bovinos provenientes de propriedades rurais com samambaia. Por outro lado, não houve alterações significativas no leucograma, indicando que não havia em nenhuma das duas categorias, resposta inflamatória sistêmica.
\end{abstract}

PALAVRAS-CHAVE: Hematologia Veterinária. Hematúria Enzoótica. Urinálise.

\section{COMPARATIVE LABORATORY RESULTS OF FEMALE NELORE CATTLE in DIFFERENT FARM CATEGORIES WITH AND WITHOUT THE PRESENCE OF Pteridium arachnoideum IN PASTURES}

\begin{abstract}
Pteridium arachnoideum, previously classified as Pteridium aquilinum, popularly known as bracken fern, is one of the most preoccupying poisonous vegetables in several countries, including Brazil. Brazilian researchers experimentally characterized three clinical forms of diseases caused by bracken fern in cattle: bleeding diathesis, bovine enzootic hematuria and squamous cell carcinoma in the upper digestive tracts. This work studies the hematological, biochemical and urinalysis values of two different phases of the animal development, being 58 heifers and 55 cows from farms with similar zootechnical characteristics, with and without the presence of bracken fern. In this study, there was a fall in the number of red blood cells in the cows and heifers from farms with the presence of bracken fern. The number of platelets was significantly higher in all of the age groups of animals from farms with bracken fern. There were no significant changes in the leucocyte count, indicating there was no systemic inflammatory response in any of the two categories.
\end{abstract}

KEYWORDS: Enzootic Hematuria. Urinalysis. Veterinary Hematology.

\section{RESULTADOS DE LABORATORIO COMPARATIVOS EN HEMBRAS NELORE DE DIFERENTES CATEGORÍAS PROVENIENTES DE GRANJAS CON Y SIN LA PRESENCIA DE Pteridium arachnoideum EN PASTAJES}

RESUMEN: El Pteridium arachnoideum, previamente clasificado como Pteridium aquilinum, popularmente conocido como helecho, es una de las plantas tóxicas más preocupantes en varios países, entre ellos Brasil. En el estado de Paraná hay varias regiones en las que la planta tiene característica endémica. Investigadores brasileños han caracterizado, experimentalmente, tres formas clínicas de la enfermedad causada por el helecho en la especie bovina: diátesis hemorrágica, hematuria enzoótica de ganado y carcinomas de células escamosas del tracto digestivo superior. En este trabajo se ha estudiado los valores hematológicos, bioquímicos y análisis de orina en dos etapas diferentes del desarrollo animal, compuesto por 58 novillas y 55 vacas, pertenecientes a granjas con características zootécnicas similares y con presencia o no de helecho. El objetivo ha sido establecer un protocolo para la detección y adoptar medidas para mejorar la gestión, evitando pérdidas. En este estudio se observó una disminución en el número de hematíes de vacas y novillas de propiedades rurales con la presencia de helecho. El recuento de plaquetas fue significativamente mayor en ambas edades de bovinos provenientes de propiedades con helecho. Por otro lado, no hubo cambios significativos en el recuento de glóbulos blancos, señalando que no había en ninguna de las dos categorías, respuesta inflamatoria sistémica.

PALABRAS CLAVE: Análisis de orina. Hematología Veterinaria. Hematuria Enzoótica.

DOI: https://doi.org/10.25110/arqvet.v19i1.2016.5784

${ }^{1}$ Universidade Estadual de Londrina, Acadêmico de Medicina Veterinária, Londrina, Paraná, Brasil.

${ }^{2}$ Universidade Estadual de Londrina, Docente, Departamento de Clínicas Veterinárias, Londrina, Paraná, Brasil. Endereço para correspondência: andrehiroshiw@hotmail.com e wilmar@uel.br. 


\section{Introdução}

A Pteridium arachnoideum, anteriormente classificada como Pteridium aquilinum e popularmente conhecida por samambaia, é um dos vegetais tóxicos mais preocupantes em vários países do mundo, incluindo o Brasil. Por causar enfermidades que acometem rebanhos de bovinos (TOKARNIA et al., 1979; NIERO et al., 1991; TOKARNIA et al., 2000), tem sido considerada a planta tóxica que causa o maior prejuízo à pecuária bovina paranaense, ocorrendo em 105 municípios (POLACK, 1990), dos 399 existentes (FERREIRA, 1996). Todavia, acredita-se que há muito mais municípios com a presença endêmica da planta (HIGASHI et al., 2014). Embora em algumas fazendas as pastagens sejam renovadas, ainda assim, persiste a samambaia, em proporções que variam de, no mínimo, brotos quase imperceptíveis a arbustos bem desenvolvidos (OLIVEIRA et al., 1998).

Jean-Blain et al. (1987) descreveram que a modificação mais importante observada é a anemia, que regride em quinze dias após cessar a hematúria. Todavia, é importante destacar que na planta há um princípio tóxico que causa também anemia aplástica, ou seja, afeta a medula óssea produtora de novas células sanguíneas. Essa constatação é bem conhecida pelos estudiosos e limita o tratamento e recuperação de bovinos acometidos, especialmente vacas.

Hayashi (1981) relatou que alterações hematológicas em bovinos intoxicados, revelaram depressão da atividade da medula óssea com trombocitopenia e leucopenia, sendo que a diminuição na contagem plaquetária estava associada à diminuição na contagem de megacariócitos, comprovada pela punção de medula óssea.

A demonstração clínica de hematúria e a aplasia medular em vacas tem sido um grande desafio para pesquisadores, pois além de cessar a urina sanguinolenta, é preciso recuperar, rapidamente, a produção de novas células sanguíneas. Por essa razão, é evidente a susceptibilidade a doenças oportunistas (PINTO et al., 2004), causando emaciação (MARÇAL et al., 2001), originadas pela trombocitopenia e leucopenia (HOPKINS, 1987; CAMPO et al., 1994; SMITH et al., 2000), mesmo que clinicamente só exista a microhematúria (RAJENDRAN et al., 1983).

Nas áreas endêmicas com presença da Pteridium arachnoideum, a urinálise tem sido utilizada como método de triagem para se estabelecer protocolo terapêutico, atualmente incluindo fitoterápicos (HIGASHI et al., 2014). Essa metodologia tem sido também fundamental para se melhorar o manejo alimentar de vacas intoxicadas pela planta para se evitar abortamentos (MARÇAL et al., 2001), o que pode ocorrer com reativa frequência em vacas intoxicadas.

Já em 1987, Hopkins comparando animais com e sem hematúria, verificou que $8 \%$ dos animais, aparentemente, sadios apresentavam eritrócitos no sedimento urinário, sugerindo que esse fato representaria um estágio pré-clínico da Hematúria Enzoótica Bovina (HEB). O volume globular, o número de hemácias, a concentração de hemoglobina e a contagem de leucócitos, não apresentaram alterações significativas quando comparado com vacas não afetadas pela hematúria.

Singh et al. (1972) estudaram as alterações hematológicas e bioquímicas de bovinos com HEB e verificaram considerável redução no volume globular e hemoglobina, com anemia progressiva, devido à perda contínua de sangue pela urina. Moderado grau de linfocitose e neutropenia foi observado, devido à condição inflamatória crônica. Diminuição dos níveis de cálcio e fósforo séricos e valores aumentados da creatinina sérica.

Ghuergariu et. al. (1990) relataram algumas alterações hematológicas e bioquímicas do sangue e urina em vacas em uma área de HEB. As análises sanguíneas e séricas dos animais com hematúria revelaram anemia e aumento da gamaglutamiltransferase (GGT), de cálcio e magnésio, porém nível normal para fósforo.

Segundo Kaneko et al. (2008) o valor de albumina inferior nos grupos doentes tem dois prováveis motivos: prenhez e lactação em comparação com animais com HEB. De acordo com os autores esses achados são importantes ferramentas de triagem em estudos comparativos.

Falbo et al. (2005) não encontraram alterações significativas no eritrograma e o leucograma de animais com hematúria "subclínica" em seu experimento. Porém, observaram hipocalcemia, nível normal de fósforo e proteinúria nos animais doentes. Neste estudo foram avaliados bovinos nascidos e criados no Estado do Paraná.

Sanchez Villalobos et al. (2006) encontraram anemia, hipoproteinemia e hipoalbuminemia, além de valores baixos de potássio, cálcio e fósforo para as vacas com HEB, destacando a forte relação tóxica com a aplasia medular.

Este trabalho teve como objetivo avaliar os valores de hemograma, bioquímica clínica e urinálise, em fêmeas da raça Nelore em diferentes faixas etárias expostas e não a planta Pteridium arachnoideum.

\section{Material e Métodos}

Para a realização da pesquisa, procedeu-se um ensaio laboratorial com amostras de sangue e urina de dois grupos de bovinos da raça nelore, criados em duas propriedades rurais com características zootécnicas semelhantes, com pastagens compostas por Brachiaria decumbens e fornecimento de sal mineralizado à vontade em cochos. Cada animal constituiu uma repetição dentro do delineamento experimental, observando assim, as diferenças dentro de cada categoria, em propriedades rurais com e sem a presença de samambaia. A pesquisa recebeu análise e aprovação do Comitê de Ética e Experimentação Animal (CEEA) da Universidade Estadual de Londrina, sob processo de número 17142/2015.

O primeiro grupo, composto por animais sadios nascidos e criados em propriedade rural isenta de samambaias (controle), localizada no município de Londrina - Paraná com altitude de 585 metros, Latitude de 2319'11'S Longitude de 51 $01^{\circ} 12^{\prime \prime} \mathrm{W}$ (GUIA RODOVIÁRIO QUATRO RODAS, 2015). O segundo grupo foi composto por animais manifestando hematúria ou microhematúria, nascidos e criados em propriedade rural infestada por samambaia, localizada no município de São Jerônimo da Serra - Paraná, com altitude de 976 metros, Latitude de $23^{\circ} 43^{\prime} 06^{\prime \prime}$ S Longitude de 5043'48' W, (GUIA RODOVIÁRIO QUATRO RODAS, 2007), local com elevadas prevalências de casos clínicos de HEB.

Para isso, foram avaliadas duas fases distintas do desenvolvimento da raça, sendo primeira fase: novilhas (15 a 24 meses de idade) e segunda fase: vacas (com mais de 36 meses de idade). Foram utilizadas 26 vacas saudáveis e 29 doentes; 29 novilhas saudáveis e 29 doentes, respectivamente.

Para realização do hemograma foi colhido cinco 
mililitros de sangue pela veia coccígena média, por meio de agulhas descartáveis e tubos comerciais a vácuo contendo EDTA, sendo o exame laboratorial realizado no mesmo dia da colheita.

Para a bioquímica clínica, colheu-se $10 \mathrm{~mL}$ de sangue, por meio de agulhas descartáveis e tubos comerciais a vácuo contendo heparina $(5000 \mathrm{UI} / \mathrm{mL})$. Para mensuração de cálcio, magnésio, fósforo e fosfatase alcalina utilizou-se o método cinético, sendo todas as amostras determinadas no equipamento automático Airone 2000.

Foram colhidas amostras de $10 \mathrm{~mL}$ de urina, durante micção espontânea estimulada por leve massagem vulvar, após higiene com papel toalha descartável. As amostras foram submetidas a exame físico, químico e análise do sedimento. No exame físico caracterizou-se o volume, a cor, aspecto, odor e densidade. $\mathrm{O}$ exame químico fez-se por meio de tiras reagentes comerciais, analisando-se o conteúdo de proteína, urobilinogênio e hemoglobina. A análise do sedimento urinário foi realizada para pesquisa de microhematúria, detectada pela presença de eritrócitos na urina (MARÇAL et al., 2002).

As urinálises foram realizadas seguindo a técnica descrita por Addis, citada por Levinson e McFate (1969) e Vallada (1981). As amostras foram centrifugadas a 1800 rpm durante cinco minutos, desprezando-se o sobrenadante $(9 \mathrm{~mL})$, restando um mililitro de urina mais os sedimentos. Foi realizada em seguida, a homogeneização e o preenchimento da câmara de Neubauer e realizada, utilizando microscópio óptico em aumento de $400 \mathrm{X}$, a contagem das células presentes no quadrante central da câmara (MARÇAL et al., 2001).

Para a realização das análises estatísticas de todos os dados foi usado o programa SAEG - Sistema de Análise Estatística e Genética, versão 8.1 da Fundação Arthur Bernardes da Universidade Federal de Viçosa, Minas Gerais.

\section{Resultados e Discussão}

A avaliação dos resultados, bem como a interpretação das análises estatísticas das duas categorias animais avaliadas, estão apresentados na Tabela 1, na qual encontram-se os valores do eritrograma. Na Tabela 2 são apresentados os valores do leucograma e na Tabela 3 os valores da bioquímica sanguínea. Os resultados da urinálise estão demonstrados na Tabela 4.
Para o grupo de novilhas e vacas, houve uma queda significativa no número de hemácias comparadas ao grupo controle (Tabela 1), tendo correlação com a presença de hematúria nas vacas (Tabela 4). O mesmo observou Jean-Blain (1987) que descreveu que o aspecto mais importante na Hematúria Enzoótica Bovina é a anemia, contrariando os resultados do trabalho de Hopkins (1987), que descreveu que volume globular, o número de hemácias e a concentração de hemoglobina, não indicaram alterações significativas quando comparado com vacas não afetadas pela hematúria.

Para os valores de volume corpuscular médio (VCM), hemoglobina corpuscular média (HCM) e concentração de hemoglobina corpuscular média (CHCM) houve diferenças significativas dentro de cada categoria entre as propriedades rurais, porém esses valores permaneceram dentro dos padrões normais.

$\mathrm{O}$ número de plaquetas foi menor estatisticamente a $1 \%(\mathrm{p}<0,01)$ nas duas diferentes faixas etárias nos bovinos provenientes de propriedades rurais com samambaia, em relação ao grupo controle. Isso poderia ser em função da perda contínua de sangue, com a tentativa de compensação dessa perda pelo animal, discordando de Hayashi (1981), que relatou que a diminuição na contagem plaquetária nos animais com toxidez pela samambaia, estava associada à diminuição na contagem de megacariócitos, comprovada pela punção de medula óssea.

Concordando com os trabalhos realizados com a samambaia em bovinos, por Smith et al. (2000) e Sanchez Villalobos et al. (2004), as alterações no leucograma permaneceram dentro dos valores de referência e as diferenças entre os grupos de animais sãos e doentes, poderia se relacionar com uma imunossupressão causada pelo metabólito Ptaquilosidio. Os resultados apresentaram-se diferentes dos avaliados por Pinto et al. (2004) que encontraram severa leucopenia e trombocitopenia em bovinos intoxicados cronicamente pela Pteridium aquilinum.

A ausência de trombocitopenia e leucopenia não nos permite sugerir a existência de uma aplasia medular comumente encontrada em animais que ingerem a Pteridium aquilinum e animais com HEB (CAMPO et al., 1994; SMITH et al., 2000). Estudos complementares poderão elucidar essa correlação. Todavia, é sabido que a Pteridium arachnoideum exerce efeitos tóxicos sobre a medula óssea, causando aplasia medular (MARÇAL et al., 2001).

Tabela 1: Médias \pm desvios padrão do eritrograma em novilhas e vacas provenientes de propriedades rurais com e sem a presença de samambaia

\begin{tabular}{|c|c|c|c|c|}
\hline \multicolumn{5}{|c|}{ Categoria } \\
\hline \multirow[b]{2}{*}{ Variável } & \multicolumn{2}{|c|}{ Novilha } & \multicolumn{2}{|c|}{ Vaca } \\
\hline & Sem Samambaia & Com Samambaia & Sem Samambaia & Com Samambaia \\
\hline $\mathrm{Ht}(\%)$ & $35,15^{*} \pm 3,60$ & $37,60 * \pm 3,95$ & $29,37 \pm 2,70$ & $31,75 \pm 7,51$ \\
\hline $\mathrm{Hb}(\mathrm{g} / \mathrm{dL})$ & $13,04 \pm 1,29$ & $12,48 \pm 1,14$ & $10,87 \pm 1,01$ & $10,16 \pm 2,81$ \\
\hline $\operatorname{He}(106 / \mu \mathrm{L}$ & $9,83 * * \pm 0,90$ & $8,73 * * \pm 0,93$ & $7,57 * * \pm 0,79$ & $6,45 * * \pm 1,48$ \\
\hline $\operatorname{VCM}\left(\mu^{3}\right)$ & $35,83 * * \pm 2,34$ & $43,22 * * \pm 2,87$ & $39,00 * * \pm 3,08$ & $48,70 * * \pm 4,70$ \\
\hline HCM (pg) & $13,21 * * \pm 0,76$ & $14,31^{* *} \pm 1,01$ & $14,37 * \pm 1,23$ & $15,59 * \pm 2,34$ \\
\hline CHCM (\%) & $37,08 * * \pm 1,05$ & $33,49 * * \pm 2,20$ & $37,00 * * \pm 1,36$ & $32,10 * * \pm 3,20$ \\
\hline Plaquetas $\left(\times 10^{3} \mu \mathrm{L}\right)$ & $76,90 * * \pm 34039,39$ & $172,20 * * \pm 56179,80$ & $70,11 * * \pm 59268,5$ & $175,86 * * \pm 102713,8$ \\
\hline
\end{tabular}


Tabela 2: Médias \pm desvios padrão do leucograma em novilhas e vacas provenientes de propriedades rurais com e sem a presença de samambaia

\begin{tabular}{lllll}
\hline \multicolumn{5}{c}{ Categoria } \\
\hline Variável & \multicolumn{2}{c}{ Novilha } & \multicolumn{2}{c}{ Vaca } \\
Linfócitos $/ \boldsymbol{\mu L}$ & Sem Samambaia & Com Samambaia & Sem Samambaia & Com Samambaia \\
Eosinófilos $/ \boldsymbol{\mu L}$ & $9809,51^{* *} \pm 2388,98$ & $7014,05^{* *} \pm 3068,05$ & $5366,01 \pm 1454,64$ & $5783,02 \pm 2486,68$ \\
Monócitos $/ \boldsymbol{\mu L}$ & $1109,09^{* *} \pm 214,39$ & $344,64 * * \pm 220,00$ & $660,48 * * \pm 485,55$ & $401,80^{*} \pm 421,16$ \\
Segmentados $/ \boldsymbol{\mu L}$ & $10,70^{* *} \pm 40,01$ & $112,18^{* *} \pm 98,16$ & $115,64 * * \pm 19,12$ & $3,68 * * \pm 168,96$ \\
Leucócitos $/ \boldsymbol{\mu L}$ & $5176,83^{* *} \pm 914,13$ & $2543,73^{* *} \pm 2032,55$ & $3478,63 \pm 1970,82$ & $3101,17 \pm 1497,12$ \\
\hline
\end{tabular}

Tabela 3: Médias \pm desvios padrão dos testes bioquímicos de novilhas e vacas provenientes de propriedades rurais com e sem a presença de samambaia

\begin{tabular}{|c|c|c|c|c|}
\hline \multicolumn{5}{|c|}{ Categoria } \\
\hline Variável & \multicolumn{2}{|c|}{ Novilha } & \multicolumn{2}{|c|}{ Vaca } \\
\hline Cálcio (mg/dL) & $10,35 * \pm 1,56$ & $11,28 * \pm 1,72$ & $10,90 * * \pm 1,57$ & $8.98^{* *} \pm 1,33$ \\
\hline Fósforo (mg/dL) & $7,56^{* *} \pm 1,21$ & $6,65 * * \pm 1,21$ & $5,23 * \pm 1,06$ & $6,13 * \pm 1,58$ \\
\hline Magnésio (mg/dL) & $2.51 \pm 0,51$ & $2,61 \pm 0,59$ & $2,22 * \pm 0,17$ & $117,09 \pm 41,02$ \\
\hline FA & $314,66 * * \pm 97,97$ & $240,88 * * \pm 77,78$ & $125,01 \pm 58,72$ & $117,09 \pm 41,02$ \\
\hline Albumina (mg/dL) & $3,90 \pm 0,71$ & $3,73 \pm 0,67$ & $3,65^{* *} \pm 0,70$ & $2,95 * * \pm 0,49$ \\
\hline Fibrinogênio (mg/dL) & $586,89 * * \pm 25,36$ & $462,07 * * \pm 152,16$ & $414,81 \pm 221,36$ & $375,86 \pm 204,68$ \\
\hline Proteína Plasmática (g/dL) & $7,69 \pm 1,42$ & $7,62 \pm 1,58$ & $6,95 \pm 1,33$ & $7,31 \pm 1,73$ \\
\hline
\end{tabular}

Tabela 4: Médias \pm desvios padrão de urinálise em novilhas e vacas provenientes de propriedades rurais com e sem a presença de samambaia

\section{Categoria}

\begin{tabular}{lllll}
\hline \multicolumn{5}{c}{ Categoria } \\
Variável & \multicolumn{3}{c}{ Novilha } & \multicolumn{1}{c}{ Vaca } \\
$\mathrm{pH}$ & Sem Samambaia & Com Samambaia & Sem Samambaia & Com Samambaia \\
Densidade & $8,93 \pm 0,22$ & $9,00 \pm 0,00$ & $9,00^{*} \pm 0,00$ & $7,96^{* *} \pm 0,54$ \\
Proteína $(\mathrm{mg} / \mathrm{dL})$ & $1012,69 \pm 8,66$ & $1019,24 \pm 20,10$ & $1012,00 \pm 8,61$ & $1016,13 \pm 13,82$ \\
Hemáceas $\left(\mathrm{x} 10^{3} / \mathrm{mL}\right)$ & $1,41^{*} \pm 1,26$ & $0,69 * \pm 0,89$ & $1,55 \pm 1,12$ & $1,79 \pm 1,14$ \\
Leucócitos $\left(\mathrm{x} 10^{3}\right)$ & $0,20 \pm 0,55$ & $202,82 \pm 1076,59$ & $0,18^{* *} \pm 0,68$ & $39043,24 * * \pm 78504,83$ \\
Células da Bexiga & $0,34^{* *} \pm 0,48$ & $1,62^{* *} \pm 1,59$ & $0,70 * * \pm 1,35$ & $15,93 * * \pm 24,09$ \\
\hline
\end{tabular}

Médias seguidas de * na mesma linha para cada categoria diferem pelo teste $\mathrm{F}$ a $5 \%$ de probabilidade $\mathrm{e}^{* *}$ diferem pelo teste a $1 \%$ de probabilidade.

Houve uma diferença significativa para os valores de cálcio com valores maiores do mineral no grupo controle, mas os mesmos se encontram dentro da normalidade $(8,0$ 12,4 mg/dL) segundo Jain (1986).

Os valores séricos de fósforo apresentaram-se inferiores no grupo provindo de propriedades rurais com a presença de samambaia para a categoria de novilhas, concordando com os valores encontrados por Singh et al. (1972) e Ghergariu (1990) que verificaram hipofosfatemia e hipomagnesemia em animais com hematúria. Contrariamente, e discordando dos valores encontrados por Rajendran (1983) e Falbo et al. (2005) que observaram valores normais para fósforo sérico em animais com HEB.

Os valores baixos de cálcio e fósforo séricos têm sido descritos associados à má absorção e filtração glomerular deficiente em animais com HEB (MENGUI et al., 2001; KANEKO et al., 2008). Falbo et al. (2005), em seu trabalho, também encontraram valores de cálcio abaixo dos valores normais para animais com HEB.

$\mathrm{Na}$ presente pesquisa, os valores de fosfatase alcalina diferiram entre as propriedades rurais para as novilhas, porém apresentaram-se dentro dos valores de referência (0196 U/L) descritos por Kaneko et al. (1997). Por outro lado, os valores de albumina foram maiores nas vacas do grupo controle. É conveniente enfatizar que alguns dos motivos da diminuição da albumina podem ser decorrentes da prenhez e lactação que, ao que sabe, causam diminuição dos valores de albumina e aumentam os níveis de globulinas durante a gestação, bem como, podem determinar perdas de albumina por disfunções renais ou lesões vesicais (KANEKO et al., 1997).

Com relação à urinálise, de acordo com Sanchez Villalobos et al. (2006) o método de tiras reagentes, utilizado nesse experimento, é de alta sensibilidade e excelente especificidade. Segundo os autores, o método de exame microscópico de sedimento urinário mostra alta capacidade de classificar um animal com HEB. Ambos os exames utilizados 
segundo os autores mostram fidelidade analítica para se detectar animais doentes.

Na presente pesquisa, a urina dos animais estudados apresentou variação no pH de 7,96 a 9,0, ocorrendo diferença significativa no grupo de vacas. Contudo, esses valores estão em concordância com os resultados encontrados por Jean-Blain et al. (1987), que realizou experimento com bovinos cronicamente intoxicados, avaliando clinicamente os resultados de urinálise.

Os grupos controle de novilhas e vacas apresentaram eritrócitos na urina, coincidindo com resultados de Hopkins (1987) que, comparando animais com e sem hematúria, verificou que oito por cento dos animais aparentemente sadios apresentavam eritrócitos no sedimento urinário.

Nas novilhas e vacas enfermas foram encontrados macro e micro hematúria, porém, no grupo das vacas o número de hemácias na urina foi significativamente maior em comparação ao grupo controle, concordando com os resultados Durão et al. (1995), cujo estudo clínico mensurou variáveis na urinálise.

Ao exame físico, a coloração da urina do grupo de animais doentes variou de amarelo róseo ao vermelho escuro, em que, a urina de alguns animais apesar de ter aspecto macroscópico normal, com coloração amarela, apresentou eritrócitos no exame de sedimento urinário, aspecto também destacado por Blood et al. (1989) em seus estudos relacionados a intoxicação por plantas em bovinos, em especial a Pteridium aquilinum.

Os valores dos leucócitos na urina foram superiores em todas as faixas etárias dentro de cada categoria, das propriedades rurais com presença de samambaia, sugerindo um processo inflamatório localizado. Ao contrário de Hopkins (1987), que em seu trabalho relatou que a contagem de leucócitos, não apresenta alterações significativas quando comparada a de vacas não afetadas pela hematúria. Porém, deve-se levar em consideração o método de colheita, pois na micção espontânea, pode ocorrer contaminação da urina.

\section{Conclusão}

As análises do eritrograma e urinálise representaram um grande suporte para o estudo e diagnóstico da HEB, porém os valores de leucograma e bioquímicos não mostraram diferenças significativas para tal diferenciação de animais com e sem HEB.

No eritrograma os valores de hemoglobina e número de hemácias, nas diferentes categorias, tiveram uma visível queda dando assim um grande auxílio na diferenciação entre as propriedades rurais.

Ainda, a urinálise serviu para caracterizar bovinos com e sem HEB, mostrando animais com presença e ausência de hemácias e leucócitos na urina, aspecto fundamental na triagem de bovinos enfermos pela samambaia, com auxílio ao diagnóstico clínico a campo.

\section{Referências}

BLOOD, D. C.; HENDERSON, J. A.; RADOSTITS, O. $M$. Disease caused by poisonous plants. Veterinary Medicine, London, 1989, c. 32, p. 1320-1322.

CAMPO, M. S. et al. Latent papillomavirus infection in cattle. Research in Veterinary Science, London, v. 56, n. 2, p.151-157, 1994.

DURÃO, J. F. et al. Aspectos anatomopatológicos e clínicos da hematúria enzoótica dos bovinos. Revista Portuguesa de Ciências Veterinárias, Lisboa, v. 5, n.1, p.11-20, 1995.

FALBO, M. K. et al. Alterações hematológicas, bioquímicas, urinárias e histopatológicas na intoxicação natural em bovinos pela samambaia (Pteridium aquilinum) (L.) Kühn. Semina, Londrina, v. 26, n. 4, p. 547-558, 2005.

FERREIRA, J. C. V. O Paraná e seus municípios. Maringá: Memória Brasileira, 1996.

GHUERGARIU, S.; BALE, G.; OROS. N. A. Unele modificari hematologice, biochimice sanguine si urinare la taurine intr-o-zona de hematurie enzootica. Revista de Zootehnie Si Medicina Veterinária, Bucuresti, n. 5-6, p.15-23, 1990.

GUIA rodoviário quatro rodas. Disponível em: $<$ http:// www.guia4rodasrodoviariopro.com.br/oque.asp $>$. Acesso em: 10 set. 2015 .

HAYASHI, T. Aplication of jamshidi's needle to bone narrow biopsy and diagnosis of bracken poisoning in cattle. Journal of The Japan Veterinary Medical Association, Tokyo, v. 34, p. 206-211, 1981.

HIGASHI, D. F.; VANZO, D. de L.; MARÇAL, W. S. Alternativa fitoterápica para bovinos com hematúria enzoótica. ANAIS DO ENCONTRO DE ENSINO, PESQUISA E EXTENSÃO - ENEPE. Anais... Presidente Prudente, p. 778, 2014.

HOPKINS, N. C. G. Enzootic haematurie in Nepal. Tropical Animal Health Production, Edinburgh, v. 19, p. 159-164, 1987.

JAIN, N. C. Cattle: normal hematology with comments on response to disease: shalm s veterinary hematology. 4. ed. Philadelphia: Lea \& Fabiger, 1986. p. 178- 207.

JEAN-BLAIN, C.; GASTELLU, J.; BRINGUIER. P. P. Hèmaturie chronique des bovines: etude clinique. Le Point Vétérinaire, Maison Alfort, v. 19, n. 106, p. 317-323, 1987.

KANEKO, J. J. HARVEY, J. W.; BRUSS, M. L. Clinical biochemistry of domestic animals. 5. ed. San Diego: Academic Press, 1997.

KANEKO, J. J.; HARVEY, J. W.; BRUSS, M. L. Clinical biochemistry of domestic animals. 6. ed. San Diego: Academic Press, 2008.

LEVINSON, S. A.; McFATE, R. P. Clinical laboratory diagnosis. 7. ed. Philadelphia: Lea \& Febiger, 1969.

MARÇAL, W. S. et al. Ocorrência de intoxicação aguda em bovinos pela samambaia (Pteridium aquilinum, L. Kuhn) no 
norte do Paraná - Brasil. Semina, Londrina, v. 22, n. 2, p. 139-144, jul./dez. 2001.

MARÇAL, W. S. et al. Intoxicação aguda pela samambaia (Ptedirium aquilinum, L. Kuhn), em bovinos da raça Aberdeen Augus. Archives of Veterinary Science, Curitiba, v. 7, n. 1, p. 77-81, 2002.

MENGI, A. K. et al. Hematological and biochemical observations on the blood of cattle suffering from chronic bovine haematuria. Indian Veterinary Journal, Madras, n.78, p. 994-996, 2001.

NIERO, L. et al. Surto de intoxicação aguda em bovinos pela ingestão de samambaia (Pteridium aquilinum, L. Kuhn) no norte do Paraná. In: SIMPÓSIO DE ESTAGIÁRIOS DO CCB/UEL, 9., 1991, Londrina. Anais... Londrina: UEL, 1991. p. 90.

OLIVEIRA, G. P.; MATSUMOTO, T.; PRIMAVESI, A. C. Ocorrência de intoxicação causada por samambaia (Pteridium aquilinum) na região nordeste do Paraná. Comunicado Técnico Embrapa, São Carlos, n. 20, p.1-9, out.1998.

PINTO, C. et al. Bovine enzootic haematuria on Sao Miguel Island - Azores. Poisonous plants and related toxins, Wallingford, p. 564-574, 2004.

POLACK, E. W. Toxicidade da pteridium aquilinum no Estado do Paraná. 1990. Dissertação (Mestrado) Universidade Federal do Paraná, Curitiba. 1990.

RAJENDRAN, M. P.; CHENNAKESAVALU, M.; NARAYANARAO, C. V. Experimental production of enzootic bovine hematuria with bracken fern. Indian Veterinary Journal, Madras, v. 60, n. 3, p. 173-178, 1983.

SANCHEZ, VILLALOBOS, A. et al. Validity, accuracy and coefficient of probability of the test urine reactive strips and microscopic examination of the urinary sediment in the diagnosis of bovine enzootic hematuria. Revista Cientifica, Facultad de Ciencias Veterinárias, Maracaibo, v. 16, n. 6, p. 604-612, 2006.

SANCHEZ VILLALOBOS, A. et al. Clinical and pathological observations in cows with Bovine Enzootic Hematuria. Revista Cientifica, Facultad de Ciências Veterinárias, Maracaibo, v. 14, n. 4, p. 317-323, 2004.

SINGH, A. K.; JOSHI, H. C.; RAY, S. N. Studies on bovine hematurie. I. Haematological and biochemical observations on the blood of catlle suffering from hematuria. Indian Journal of Animal Science, New Delhi, v. 43, n. 4, p. 296299, 1972.

SMITH, B. L. et al. Helechos: la toxicidad animal y la salud humana. La Habana: Memorial, 2000. p. 2-11. (Curso Internacional de Toxicosis por Plantas).
Plantas tóxicas do Brasil: Região Sul. Rio de Janeiro: Universidade Federal Rural do Rio de Janeiro, 1979.

TOKARNIA, C. H.; DOBEREINER, J.; PEIXOTO, P. V. Plantas tóxicas do Brasil. Rio de Janeiro: Helianthus, 2000. p.178-187.

VALLADA, E. P. Exame quantitativo do sedimento urinário. Manual de exames de urina. 3. ed. Rio de Janeiro: Atheneu, 1981. cap. 6, p. 89-95.

TOKARNIA, C. H.; DOBEREINER, J.; BARROS, S. S. 